\title{
LOG-IDEAH: LOGic trees for identification of damage due to earthquakes for architectural heritage
}

\author{
V. I. Novelli · D. D’Ayala
}

Received: 18 July 2013 / Accepted: 7 April 2014 / Published online: 9 May 2014

(C) The Author(s) 2014. This article is published with open access at Springerlink.com

\begin{abstract}
In the framework of PERPETUATE, LOG-IDEAH: "LOGic trees for Identification of Damage due to Earthquakes for Architectural Heritage" has been developed as a post-earthquake assessment tool for the evaluation of the global seismic performance of architectural assets (AAs). LOG-IDEAH is an expert system which interprets the seismic damage collected on site by relying on the knowledge of engineers and architects in seismic vulnerability assessment. The present expert system has been set up on logic trees, implemented in answer set programming to encode the recognition process that surveyors proceed to investigate the causes of damage and to evaluate the failures occurring on an AA. LOGIDEAH, available at (http://perpetuate.cs.bath.ac.uk/), allows sketching AAs, recording the state of damage, uploading GPS referenced images and comparing collapse mechanisms observed on site with collapse mechanisms identified by the system. The seismic damage, which is the minimum data required by LOG-IDEAH, is collected at the level of both structural elements and artistic assets (local level) and interpreted at the level of the AA (global level). The answer set programming applied for the construction of the machine-processable representation, once the input data has been recorded into the web-interface, provides all possible failure modes and related possibilities of occurrence by taking into consideration the reliability level of the collected data. Its capability has been validated by applying LOGIDEAH to the historic centre of L'Aquila of the 2009 earthquake by pointing out that answer set programming is a valid alternative approach to the existing methods developed to identify the seismic performance of masonry AAs.
\end{abstract}

Keywords Semantic expert system - Answer set programming - Masonry historic architectural assets · Damage scenarios · Post-earthquake assessment · Global mechanisms

\footnotetext{
V. I. Novelli $(\varangle) \cdot$ D. D’Ayala

University College London, LondonWC1E 6BT, UK

e-mail: viviana.novelli@ucl.ac.uk

D. D'Ayala

e-mail:d.dayala@ucl.ac.uk
} 


\section{Introduction}

During a post-earthquake assessment, engineers and architects are responsible to identify the level of safety of AAs on the basis of crack patterns observed on site and to recommend, if necessary, restraining interventions for preventing further damage. Sometimes the restricted time to perform surveys after an earthquake due to limited access to damaged area might imply that the identification of the structural performance of AAs relies on partial inspections. Since many engineers and architects who offer their contribution as volunteers for post-earthquake assessments do not always have ample experience of this type of survey, several countries have developed guidelines: California (ATC20 and ATC20i; ATC 1989; ATC 2003); Italy (DPCM 2006); Mexico (CENAPRED 1996) and Colombia (AIS 2003) are some examples. Furthermore, nowadays several approaches: AeDES (D.P.C.M. 2006, O.P.C.M n. 3753 (OPCM 2009), Medea (Zuccaro and Papa 2007) and de Vent et al.'s methodology (De Vent et al. 2008) are also available to estimate the safety of the AAs on the basis of the standards defined in the national codes. These methodologies are mainly produced with the aim of performing a screening and tagging, which is needed to ascertain whether AAs should be written off (red tag), inspected more accurately (yellow tag) or can be used right away (green tag). Further, they may be used to estimate the costs of the provisional post-earthquake interventions. For this reason, damage types and their severity are listed rather to judge the safety than to recognise the seismic performance of the AAs. Furthermore, these approaches, in order to identify the safety of an AA, often require a consistent amount of data, which needs to be collected by indoor inspection. A common issue to all these methodologies is also: the difficulty to identify the position of the damage on the AAs on the basis of the information which have been collected for these methodologies and consequently the difficulty to evaluate the type of failures occurring on the inspected structure.

Vatan and Görün (2011) propose to record the location of damage on the structural elements by defining their position with respect to a reference axial system fixed in the plan drawing of the AA under consideration. By this axial system, set by drawing axial lines along each structural element, denoted in one direction by letters in the other direction by numbers, a code for the identification of the position of the damaged elements is created. The limitation of the present method is that detailed internal inspections are required before assessing the seismic damage of an AA, since an axial reference system only can be set in the plan drawing of an AA after a good understanding of the AA plan.

Since meticulous surveys are difficult to be carried out, when large number of AAs with highly fractioned ownership are considered, and when AAs, particularly damaged by seismic events, are not inspected for safety reasons, approaches for post-earthquake assessment which require few parameters for the identification of the AA behaviours and use of devices able to share real-time information with users and other systems, are usually better favoured.

In recent years GIS-GPS systems have been intensely used for storing, elaborating, and mapping post-event spatial data in Turkey (Turker 2005), Chile (Hiroyuki et al. 2006), Iran (Hoffmann 2007), China (Tong and Liu 2009), Haiti (Corbane et al. 2011), and L'Aquila (Dell' Acqua et al. 2009). These applications, which use satellite imagery and data positioning for the identification of seismic damage point out that these systems are particularly effective to recognise AAs affected by partial or total collapses of roof and floors. This entails that for localised collapse or for level' of damage of lesser severity, it is not straightforward to attribute the correct damage state or extension (Maruyama et al. 2012; Hassanzadeha et al. 2012; Wielanda et al. 2012). The quality of assessment obtained by using satellite imagery can be improved by coupling this with aerial images, since this allows capturing of also Vertical Macro Elements which partially or totally fail for overturning. However, the use of satellite 
and aerial pictures images provide a better damage scenario only when AAs are partially or totally collapsed, roofs have failed or debris is visible. With respect to damage types of moderate severity these are still difficult to be observed with the images mentioned above, since at the present time only close up pictures of buildings are suitable to assess damage with different levels of severity. This explains why satellite images and aerial images are used by insurance companies, which only pay policyholders of houses completely damaged by seismic events, and are not used for the identification of the seismic behaviour of AAs with low damage.

The current levels of resolution and possible manipulation of satellite and aerial imagery commonly available is not suitable for the evaluation of the AA response, which is a process that requires close range vertical pictures and a minimum of data to characterise the inspected AAs. The type of pictures that should be used for a seismic assessment needs to be taken with smartphones, tablets or GPS camera which can be georeferenced into GIS system. Moreover, field pictures require high image resolution, in order to allow identifying crack patterns with different level of severity. Nowadays, the early warning systems (Kanamori 2005; Pollino et al. 2012), GPS data (Serra et al. 2013), public domain open-source software (RISe by Lang et al. (2010)) allows collecting pictures and data in real time, indeed the real issue is: how this information collected by these sophisticated tools can be adopted as a data to identify the seismic performance of AAs. The type of data needed for the identification of the seismic behaviour of an AA depends on how the seismic assessment methodologies require and process the input data on the basis of their objectives. The existing seismic assessment methodologies are categorised into two classes: numerical approaches based on empirical methods: AEDeS (Baggio et al. 2009); Simplified Index Method (Lourenco and Roque 2005) OTSO (Bosiljkov et al. 2009) and numerical approaches based on analytical methods: FaMIVE (D'Ayala and Speranza 2003; D'Ayala 2005; D'Ayala and Paganoni 2011); Vulnus; TreMURI (Lagomarsino et al. 2013). Moreover building performance can also be investigated by using predefined fragility curves (HAZUS-MH), which are often used as input of seismic risk platforms such as SELENA (Molina et al. 2010) and ELER (Hancilar). However predefined fragility curves have the limitation of being defined only for some building typologies located in specific regions.

Furthermore, the approaches mentioned above are not always able or require complex analytical models to estimate how the decay caused by not only seismic events but also by alterations not executed according to the conservation standards, affects the performance of buildings. This entails that although these weaknesses in a building are difficult to be simulated by using analytical approaches, these are fundamental and need to be taken into consideration to understand why buildings of same building typology have different performances. Field experience is also very significant to evaluate the causes of a failure in a building; however encoding the knowledge of surveyors in analytical models is not straightforward. For this reason tools based on Artificial Intelligence (AI), a branch of computer science which develops intelligent machines which think and process input data as human being, have been investigated.

In the field of seismic damage assessment of AAs AI has been applied for a number of knowledge based on systems: DASE (Melchor-Lucero and Ferregut 1995), developed for estimating damage level and failure modes of concrete AAs; as a support to neuro-fuzzy interference system: SPERIL (Ogawa and Fu 1985) implemented for the post-earthquake assessment of concrete and steel AAs. One of the most recent applications of AI is the postearthquake assessment tool EDE: Earthquake Damage Evaluation of AAs (Carreno et al. 2004). This is an artificial neural networks and fuzzy logic approach which defines the safety level of the AAs by computing a damage index as a function of the damage levels on structural 
and non-structural elements, the soil conditions and the state of the AA before the seismic event. Since this approach aims at providing the level of safety, the damage index is calibrated on the severity of damage rather than on the type of damage. AI has been also applied to implement the knowledge-based system IGOR (Cadei et al. 1990) developed with the aim of identifying the seismic risk of masonry AAs by encoding engineers and architects' expertise for the evaluation of structural behaviour. This approach requires a large number of the input data in order to estimate the possible critical scenarios for the AAs under consideration and to compute the probability of occurrence for an identified scenario. However, IGOR approach is not always able to simulate all critical scenarios, and when this happens the logic maker often chooses the most suitable interventions for the AAs in economic terms rather than structural terms (Dovers and Handmer 1995). Actually, since the existing AI tools identify the strengthening interventions on the basis of the severe damage instead of failure types occurring on the AA, it might be difficult to judge whether the interventions recommended by these systems are suitable to prevent the collapse.

For this reason, in order to identify the failure modes on AAs, in the framework of PERPETUATE, an FP7 project which provides European Guidelines for the evaluation and mitigation of seismic risk to cultural heritage assets, the authors have developed a post-earthquake assessment tool and called it LOG-IDEAH: LOGic trees for Identification of Damage due to Earthquakes for Architectural Heritage. The present software identifies failure modes of masonry agglomerates by using a declarative programming, a style of computer programming which expresses the human thinking by algorithms. The declarative programming used for LOG-IDEAH is the answer set programming (ASP), which is adopted to encode the experience used by engineers and architects in interpreting the seismic damage as collapse mechanisms. The encoding in ASP is entailed by defining set of rules which are used by the software to verify whether the seismic damage collected on site points out the occurrence of one or more collapse mechanisms. The seismic damage and geometry of the AA are the minimum requirements for LOG-IDEAH to estimate the collapse mechanisms occurring on a masonry AA. These data can be collected and stored by using web-interface available online at (http://perpetuate.cs.bath.ac.uk/). This web-platform is directly connected to the ASP; therefore once the ASP has interpreted the input data, gathered directly from the website, and the collapse mechanisms are identified, the output will be automatically displayed on the same platform, which is used for the data collection. The lower the uncertainty level of the collected data is; the higher the possibility of occurrence of a type of failure identified on site or computed by the system. For this reason, in order to take into account the level of reliability of the input data, LOG-IDEAH provides not only the type of collapse mechanism, but also the possibility level of occurrence of each failure identified by the system. The system is optimised for PCs and tablets with Windows, Android or MacOS operating systems and allows real-time data sharing, uploading GPS pictures, localising the position of the observed AA on Google Maps, recording the state of damage to buildings and comparing failure modes observed on site with failures modes computed by the ASP. Within the last three years, the database of LOG-IDEAH has been filled up with records collected for AAs in Italy, Spain, Algeria and Slovenia. The tool has been tested in the historic centre of L'Aquila in Italy, by identifying the seismic performance of the masonry AA blocks damaged by the earthquake in 2009.

In the conclusion of this paper, it is emphasised that AI approaches, used to solve complex problems by developing functions which imitate the human brain, are suitable to replicate the intuitive approach used by engineers in the identification of the seismic performance of masonry AAs. 


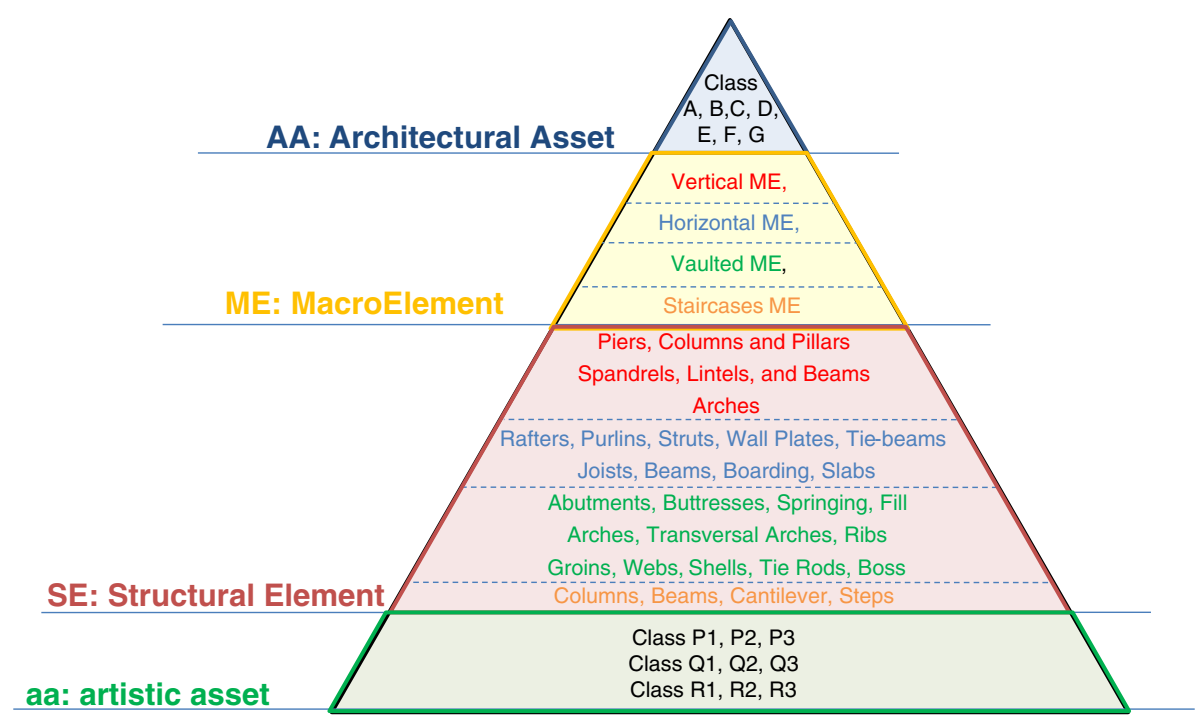

Fig. 1 Hierarchic pyramid of LOG-IDEAH. This is composed by four top levels: architectural assets, MacroElements, structural elements, and artistic assets

\section{Hierarchical structure of architectural assets}

The hierarchical approach used by the surveyors to assess the seismic performance of urban blocks entails: observing the damage at the level of the single AA and interpreting the observed damage at the level of the urban block is replicated in LOG-IDEAH defining a hierarchical approach that de-constructs the urban block in the architectural assets (AA), Architectural Asset into macro-elements (ME), the macro-elements into structural elements (SE) and the structural elements are identified as being linked to or being in itself artistic assets ( $a a)$. This implies that the performance of an urban block ensues from data collected at the level of the SEs and aas (local level) and interpreted first at the level of the ME and then at the level of the AA (global level). The transition from local to global level is discussed in Fig. 1 by defining the hierarchy among the elements of an urban block by a nomenclature which link single damaged and undamaged elements to a ME, single MEs to an AA and AAs to an urban block. The state of damage, as reported in Sect. 4, is collected by observing the damage type and the damage severity and position of the damaged at level of the SEs and aas. The evaluation of the performance at global level is carried out by the rules of Sect. 5 implemented to interpret the local damage as the response of the single structural elements to a phenomena (mechanism) occurring at the level of the AA.

The system of Fig. 1, which represents the AA as a pyramid composed of multiple levels of objects linked to each other, suits the hierarchical approach set for LOG-IDEAH. The four levels of objects captured in the pyramid in Fig. 1 are: (1) AA: this level covers seven classes of AAs: from A to G, as defined in Lagomarsino et al. (2011), from mansions, trough mosques, and churches; (2) $M E$ : this level covers four classes: Vertical ME (VeME), Horizontal ME (HoME), Vaulted ME (VaME) and Staircases ME (StME), as they are defined in D' Ayala and Novelli (2010); (3) SE: this level covers four classes, corresponding to the MEs above, such as piers and spandrels (vertical), rafters and tie beams (horizontal), abutments and arches (vaulted) and cantilever and steps (staircases), as they are defined in D'Ayala and Novelli 
(2010); (4) $a a$ : this is a set of three groups: (P: Structural elements with artistic value, Q: artistic value which is strictly connected to structural elements and R: aas which are not strictly connected to structural elements) as they are defined in Lagomarsino et al. (2011).

\section{Methodology}

\subsection{Data acquisition protocol}

Historic city centres usually have a clustered formation (or blocks or aggregates of AAs) constituted by buildings built up in different historical era. This entails that the AA typologies within a clusters may differ greatly with adjacent AAs, since they might be constructed at different times and with different techniques and layouts.

This entails that the AA typologies within a cluster may differ greatly, as well with adjacent AAs commonly being constructed at different times, possibly using different techniques and layouts. In order to take into account the influence of adjacent AAs on their seismic performance the data collection is aggregated at the level of the urban block, by recording the geometry of the AA block under consideration and the number of the AAs which belong to it. The data acquisition is performed in-situ or via detailed photography and the level of reliability for the collected information has to be explicitly recorded by the surveyor. Since post-earthquake assessments are often conducted from the street without directly entering the AA due to the unknown level of risk of collapse, and usually augmented by pictures, the data is often collected on VeME rather than on HoME, VaME and StME.

For this reason, LOG-IDEAH identifies the global seismic performance of an AA assuming that the collapse mechanisms are captured on the basis of the damage observed on piers, pillars, arches and spandrels which are (SE) or in itself (aa). Once the data collection is complete on the VeMEs, hypotheses are made on the damage state of the uninspected elements on the MEs, as it is discussed in Sect. 5.3.

The uncertainty of the AA performance captured by LOG-IDEAH is enriched or reduced by the accuracy of the assumptions made on the observed and unnoticed elements, and the data interpretation carried out first at the level of the MEs and then at the level of the AAs, according to the rules defined in the ASP of Sect. 5. This entails that in essence the survey of an AA consists of deconstructing the AA under investigation, into VEMEs which are identified according to their orientation and anticlockwise numbering; the VeMEs into SEs: piers/columns, spandrels/arches; and the SEs into aas. The relationship between AA block and AA, AA and the VeMEs is determined by identifying and numbering urban blocks on a reference urban plan, and then enumerating the AA located within each urban block, and enumerating the VeME belonging to each AA. Therefore, the name associated with the AA under investigation is given by: (AA block number + AA number), and that associated with the inspected VeMEs by adding the VeME number and orientation.

\subsection{Relationships between vertical macroelements and structural elements}

In order to create links between MEs and SEs, the VeMEs are decomposed to horizontal and vertical structural elements while a univocal system of identification for each element is provided by defining a grid on the VeME, Fig. 2 . The example in Fig. 3, which refers to VeME 10.4.1e of the AA number 4 of urban block 10 which is inspected in the historic centre of L'Aquila damaged by the 2009 earthquake, illustrates how the VeME is decomposed into Structural Elements to enable reference to pier 2 on floor 1. On the right hand side of Fig. 3, 

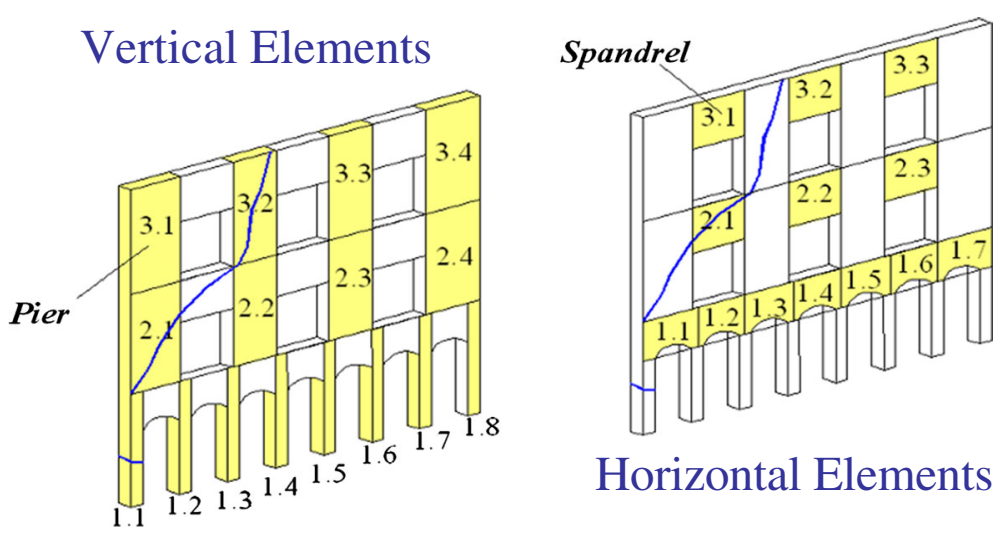

Fig. 2 Naming of the structural elements of a VeME. Two labels are associated to each vertical and horizontal element, where (nf) identifies the number of the floor and (i) the position of the element at each floor



(a)
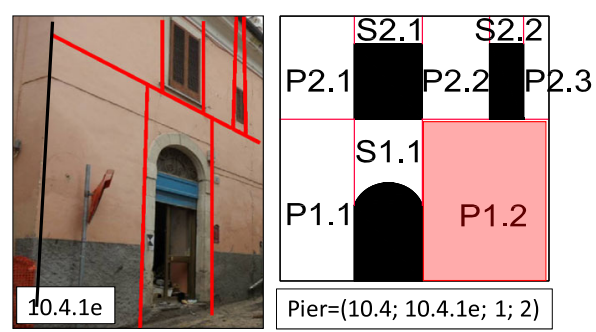

(b)

Fig. 3 a Location of the AA in the urban block and naming convention to relate urban block, AA, VeMEs and SEs which highlights that only two VeMEs have been inspected: 10.4.1e and 10.4.4s; b Deconstruction of the VeME 10.4.1e in structural elements and definition of the string which points out the position of the pier P1.2: (AAname, VeME name, (nf) of the pier in red, (i) of the pier in red)

the deconstruction of the VeME 10.4 and the denomination of the structural elements are illustrated.

\subsection{Relationships between structural elements and artistic assets}

The correlation between SEs and aas is set by defining a string, see Fig. 4, which contains the name of the inspected AA, the name of the observed VeME, the position of the SE the aa is associated to, and the name of the aa type defined according to a classification introduced in Lagomarsino et al. (2011); D’Ayala and Novelli (2010), and D'Ayala and Novelli (2012a).

\section{Local damage type and local damage level}

The structural damage types which can be observed on site are listed in Fig. 5, while the structural damage severity is classified according to Table 1, as they are defined in Eurocode 8 (CEN 2005).

For the sake of illustration, Fig. 6 (on the left) highlights the damage of the VeME 10.4sw, as it is recorded in the two strings (Pier_cracklocation) and (Spandrel_cracklocation) defined for 


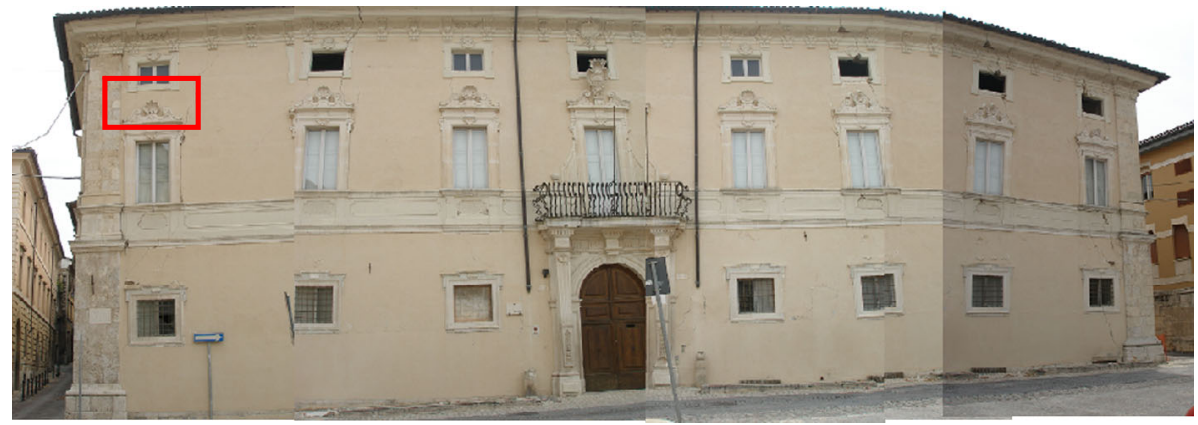

Fig. 4 Denomination associated to the aa. The string $(2.1,2.1 \mathrm{e}, 2,1$, Q2) defines the position of the SE to which the aa, highlighted in red, is attached to. Therefore Q2, which is the type of aa identified on the building in the picture, is on the first SE at the second floor of the VeMe on the east side of AA, named 2.1

\begin{tabular}{|c|c|c|c|c|c|c|}
\hline $\begin{array}{c}\mathrm{V}=\text { Vertical } \\
\text { Crack }\end{array}$ & $\begin{aligned} \mathbf{H}= & \text { Horizontal } \\
& \text { Crack }\end{aligned}$ & $\begin{array}{c}\text { D1 = Diagonal } \\
\text { Crack }\end{array}$ & $\begin{array}{c}\text { D2 = Diagonal } \\
\text { Crack }\end{array}$ & $\begin{array}{c}X=X \text { Shape } \\
\text { Crack }\end{array}$ & S = Spalling & $\begin{array}{c}\mathrm{Cr}= \\
\text { Crushing }\end{array}$ \\
\hline 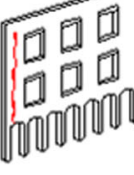 & 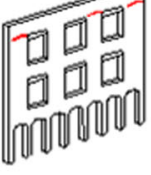 & & 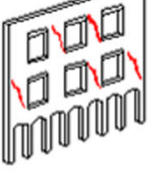 & 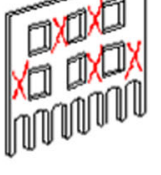 & (1) & (1) 1 \\
\hline
\end{tabular}

Fig. 5 List of structural damage types identifiable on site

Table 1 List of structural damage levels identifiable on site

LD: light damage:

$S D$ : significant damage:

$N C$ : near collapse:

$C$ : collapse:
No or slight structural damage, no or slight non-structural damage, the AA is fully operational

Moderate structural damage, moderate non-structural damage, the AA is operational

Serious structural damage, serious non-structural damage, the AA is repairable Very serious structural damage

damaged piers and damaged spandrels, respectively. In order to link damage type and damage level observed on the SEs, to the entire AA, the string includes the following information: AA name, VeME name, position of the damaged SEs, damage types of the damaged SEs, and damage level for each damage type).

As for the aas, the approach for the seismic damage collection is similar to the one applied for the SEs. The damage types and severity of damage observable on site on aas are defined in D'Ayala and Novelli (2010) and (2012a). Once the damage types and damage levels have been observed for each aa type, a similar string can be defined to store the damage for these SEs. Figure 6 points out the seismic damage observed on the aas circled in red, which is recorded in the strings (artisticspandrelCrack) defined for the damaged spandrels. The reliability level for this data is "High", since the data has been collected on site and photographic documentations are also available. 


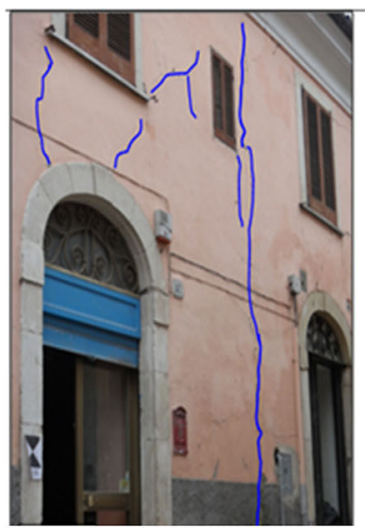

Pier_cracklocation

$=$ array with the damaged Vertical Structural

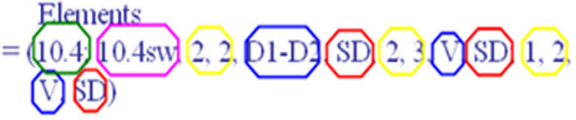

Spandrel_cracklocation

$=$ array with the damaged Horizontal Structura

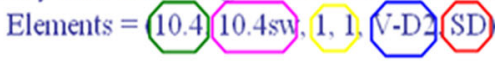

\begin{tabular}{|c|c|c|}
\hline Architectonic Asset & Facade & Damage Position \\
\hline Damage type & Damage level \\
\hline
\end{tabular}

(a)

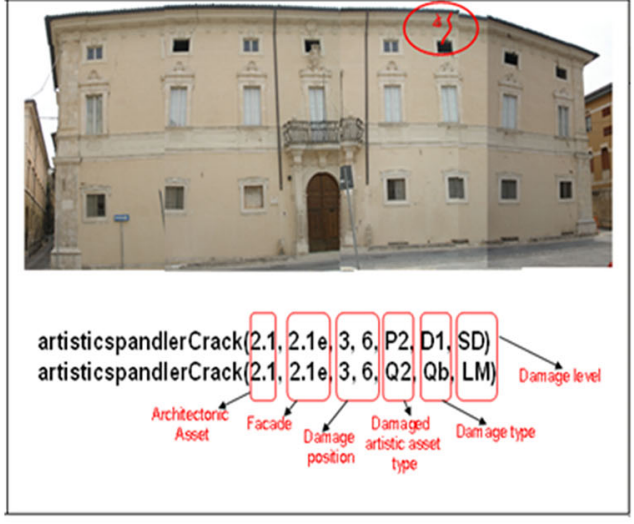

(b)

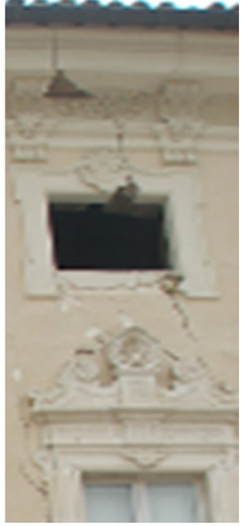

(c)

Fig. 6 a Damage identification for the SEs of the VeME 10.4sw and $\mathbf{b}$ damage identification for the aas and c closer up picture of the damage on the artistic assets

\section{Representation and reasoning}

\subsection{Logic trees}

The identification of the seismic performance of an AA is carried out by using logic trees, specified to encode the process of recognition for 18 possible collapse mechanisms, in Fig. 7 , by interpreting the seismic damage types and levels defined in Sect. 4 . The logic used in the logic trees to identify the occurrence of a collapse mechanism follows specific rules which are based on matching the seismic damage pattern observed on site and defined according to Sect. 4 with the failure modes of Fig. 7.

Some of these collapse mechanisms can be identified by using data attributed to a single VeME since they occur as failures of a single façade. For others, such as mechanism A2 and the failures belonging to the classes $\mathrm{B}$ or $\mathrm{C}$, since they represent failures which involve at least two VeMEs, they can be correctly interpreted only if the seismic damage pattern has been observed on at least two adjacent façades. In case the crack pattern observed on a 


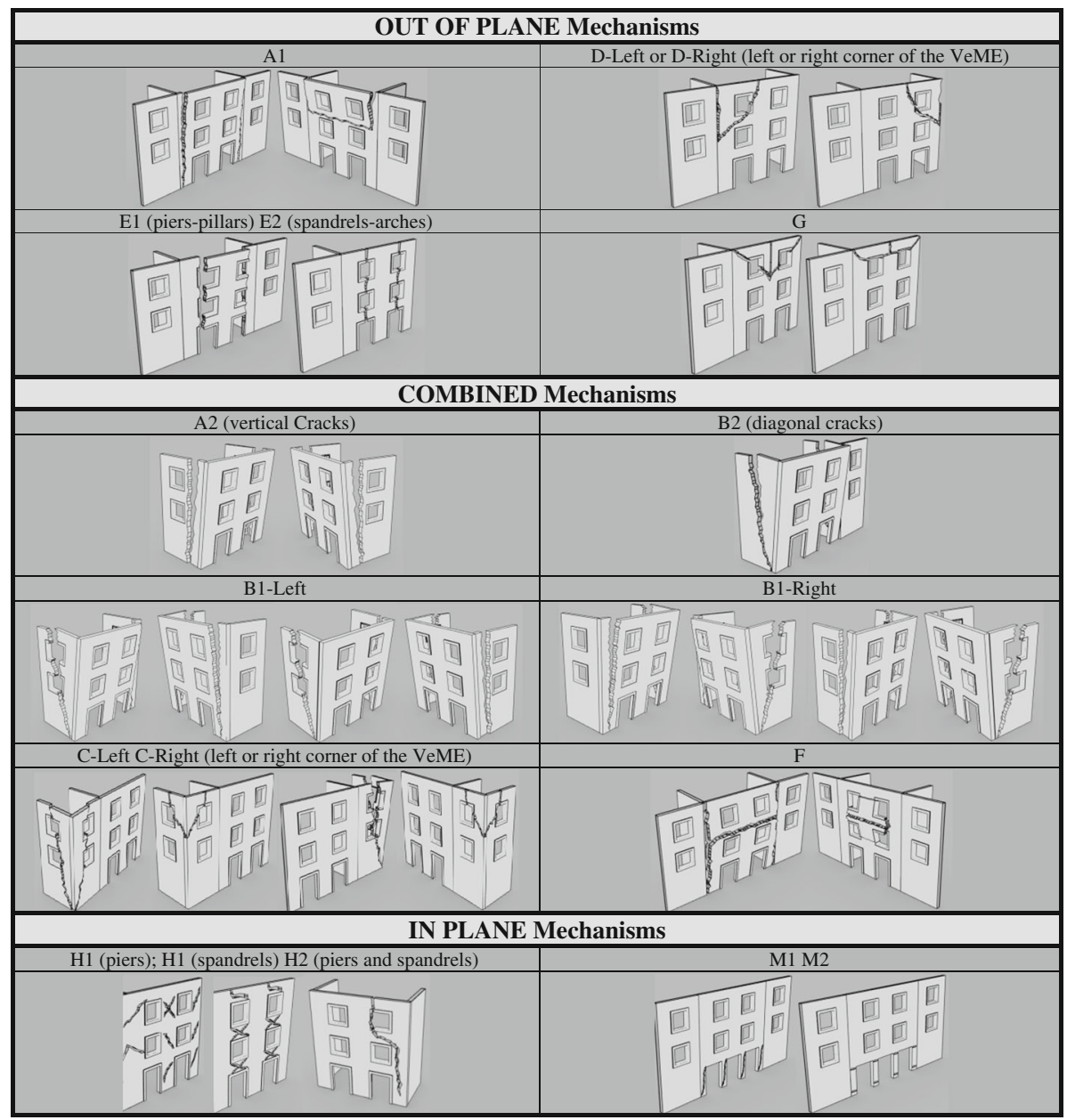

Fig. 7 Possible collapse mechanisms identifiable by LOG-IDEAH

single VeMEs or on a group of adjacent ones does not completely match which one of the collapse mechanisms defined in Fig. 7, the most plausible collapse mechanism is assigned by making certain assumptions on the damage pattern of the adjacent VeMEs which have not been directly inspected.

As the three-dimensional AA has been deconstructed in two-dimensional VeMEs to record and localise the crack pattern, in order to determine the failure mode of the whole asset, the procedure should be iteratively applied to as many groups of adjacent façades as can be determined by the surveying phase, so as to consider all possible collapse mechanisms.

The rules developed in the logic trees are in some cases mutually exclusive, in some cases possible alternatives. The mechanisms are captured by identifying conditions of weakness or strength on the VeMEs under observation which are recognized on the basis of the damage pattern observed on the façade inspected on site or by using pictures. For instance, if mechanism A1, a failure for vertical cracks is identified for a VeME, it means that such VeMe has poor connections with its adjacent VeMEs and between it and the HoMEs. 
Therefore any other mechanism, such as combined mechanisms A2, Bs or C and out of plane mechanisms D or E are excluded as possible failures, as all of them imply that the VeME under inspection has good connections with at least one of its adjacent VeMEs and/or good connections with the HoMEs. However the logic trees do not a priori exclude the identification of other mechanisms whose occurrence is not constrained by the presence of connections to other VeMEs. The effect of strengthening interventions, such as ties and roof connection are also considered in the logic trees and if they are present on the inspected VeMEs, the observed crack pattern is interpreted taking this into account to verify whether the pattern observed matches mechanisms of type F.

Given a set of input data with its level of reliability, the procedure described above produces an output of one or more mechanisms as possible interpretation of the crack pattern. At this stage the operator has two options: either to accept the identified possible mechanisms or to reject any of them and re-assess the initial set of data. In latter case the data can be re-codified either by checking the classification of the single cracks in terms of position, type and level of damage or by reducing or expanding the current set of data constituting the crack pattern under analysis and any visible strengthening provision. This would then constitute a new set of updated data for the given VeMe and corresponding AA. The logic underlying the whole procedure can be illustrated by way of the examples given in Figs. 8 and 9 which lead to the identification of A2 mechanisms. Details of the logic tree associated to the identification of each possible mechanism are provided in the Appendix of D'Ayala and Novelli (2012a).

In Figs. 8a and 9a the logic trees (DT)-Al and DT-Ar are reported, and in Fig. 8b and 9b and $9 \mathrm{c}$ the notes, which highlights on which floor the logic trees are operating at a given time are included. The logic trees (DT) $A-l$ and $D T A-r$ are developed to identify presence of vertical cracks on the VeME_R and VEME_L, by starting from the top floor to the bottom floor, as it is reported Figs. 8 and 9.

These DTs are only used in the hypothesis that no cracks have been identified on the central VeME of Figs. 8 and 9. In case the crack patterns identifiable by $D T A-r$ and $D T A-l$ are not recognised, mechanism A2 is identified as a possible failure of the central VeME, and mechanisms in class B, C, D are automatically excluded. Once A2 is recognised, DT A-1 proposes to check if mechanism $\mathrm{F}$ of Fig. 7 is occurring too.

In case a vertical crack pattern is identified only on the VeME_R or VeME_L according to the DT A-r and DT A-l, next step is to check whether mechanisms B1-Left or B1-Right are occurring as proposed in the logic trees in Figs. 8 and 9. In case B1-Left or B1-Right are not occurring, further hypotheses are checked in order to identify the possible mechanisms taking in place on the VEME under investigation, by using the observations reported in Sect. 5.3, relative to the assessment of the data quality and completeness. In case the vertical crack patterns analysed by the $D T A-r$ and $D T A-l$ do not lead to the identification of A2, then the procedure assesses the possible occurrence of mechanisms belonging to class $\mathrm{B}$. As described so far, the use of the traditional logic tree would entail reassessing the same data through a large number of consecutive iteration which would progressively exclude the least likely results so as to produce the most probable mechanism, i.e. the one or ones that best match the observed crack pattern. In order to reduce this computational burden and best mimic the intuitive process that the operator will go through, it has been chosen to use the Answer Set Programming environment, as explained in detail in the next section.

\subsection{Answer set programming for the identification of the collapse mechanisms}

As illustrated in Sect. 5.1 the data entry to the logic trees is a phenomenological character rather than an epistemological character. In other words having observed a phenomenon, 


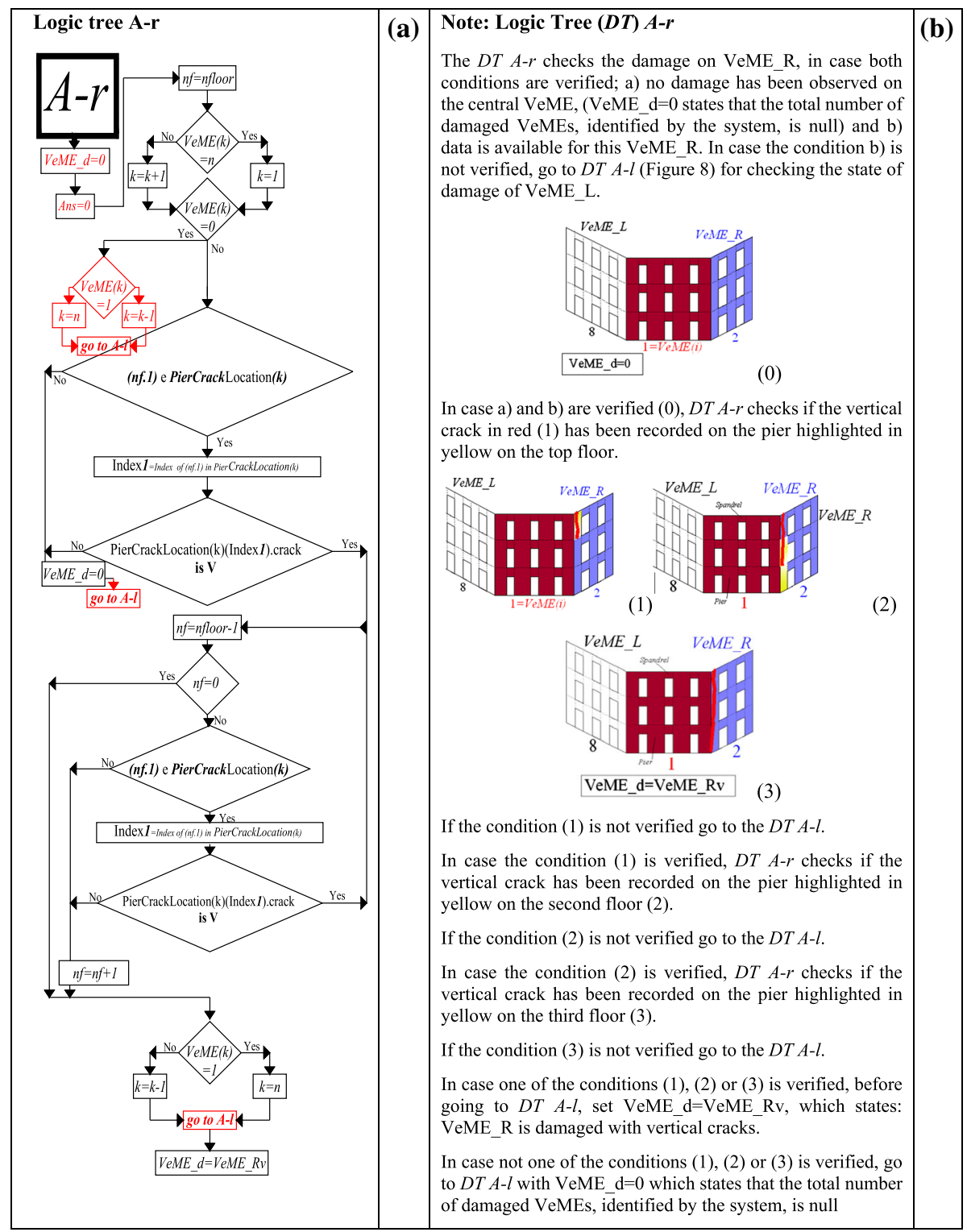

Fig. 8 a Logic tree A-r for the identification of the vertical crack pattern on the VeME_R and $\mathbf{b}$ Note, which highlights on which floor the logic trees are operating at a given time

the mechanism, and having established a logical correlation between its occurrence and a set of evidences, (distribution of cracks, their entity and presence of strengthening devices, we can use the set of evidences as a proxy to compute the possibility of occurrence of the phenomenon under scrutiny.

This implies that the logic trees developed in the previous Section determine the set of rules for the evidence that is collected to match the phenomenon sought, rather than use in the mechanical attributes of the structures which are causative of that phenomenon. Such logic 


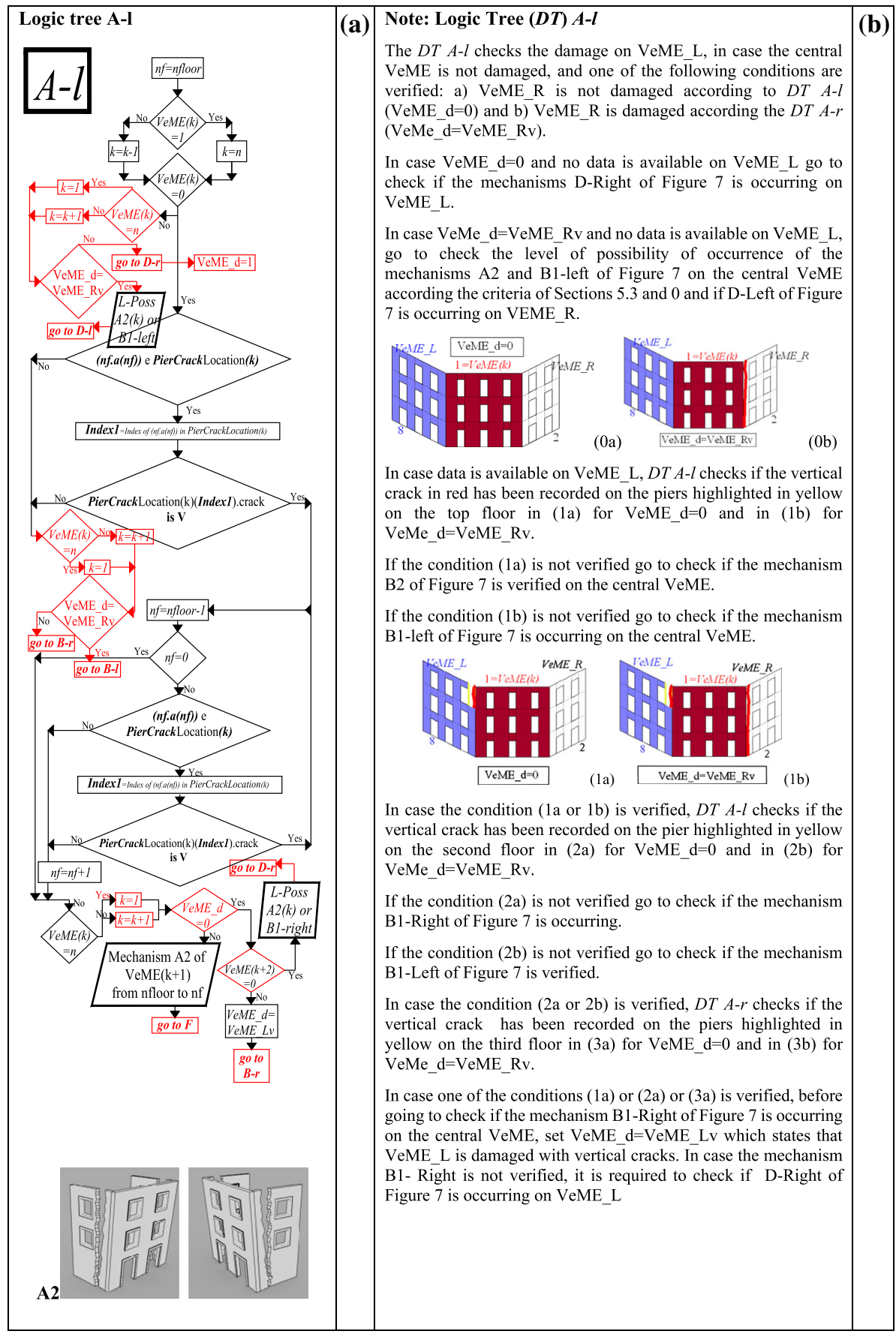

Fig. 9 a Logic tree A-1 for the identification of the vertical crack pattern on the VeME_L and b and $\mathbf{c}$ Note, which highlights on which floor the logic trees are operating at a given time 


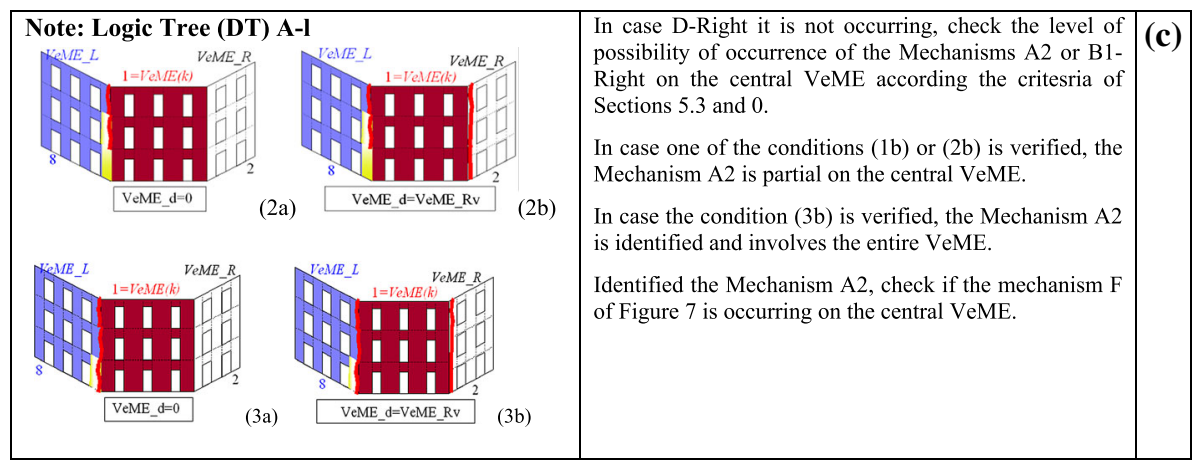

Fig. 9 continued

can be defined as declarative semantics (Wuwongse et al. 2001; Brain et al. 2009). Although the traditional logic trees can be useful to establish the links between a large set of phenomena and specific occurrences to a particular mechanism, they are nested and cumbersome from the point of view of programming.

Furthermore, since the structure of the logic trees imposes that the various conditions, leading to a specific occurrence, are nested and mutually exclusive, they do not reflect the reality where a set of data might lead to different occurrences.

This situation is particularly suitable to be treated with an answer set programming (ASP) approach (Gelfond and Lifschitz 1988; Gelfond and Lifschitz 1991).

In this specific application AnsProlog (Chitta 2003) is the chosen implementation language used to write the logic code to define the rules which describe the collapse mechanisms of Fig. 7 by their crack patterns.

Since the ASP is directly integrated with a web-interface (Sect. 6) which records and stores the geometric information and seismic damage for single AAs in XML file format, the ASP develops the representation and performs the analysis of a single AA per time. This is achieved by defining semantic tags which allows linking the XML data structure gathered through the web-site according to the hierarchical approach used to deconstruct the AA as described in Sect. 2. As it is pointed out in Fig. 10, which contains the encoding of the data for a single VeME with vertical cracks on its edges, it can be seen that the format of each line of the AnsProlog code corresponds to the string format introduced in Sect. 4 and Fig. 6, matching the same deconstructive process highlighted above.

Figure 11 shows the AnsProlog code which is able to detect the collapse mechanism A1 on the basis of the information stored in Fig. 11. Most of the encodings of the collapse mechanisms, and mechanism A1 is not an exception, start from the top floor of a VeME and try to identify a certain crack pattern. If found, lower floors are tested until a floor is found which does not have the desired pattern. The system will then return the specific pattern with the range of floors involved in the pattern (Fig. 12).

For the encoding, since distinct parts of a required crack pattern are implemented separately (e.g. patternAr and patternAl), these can be reused for encoding parts of crack pattern of different collapse mechanisms.

For the sake of comprehension, Fig. 13 has been reported to illustrate the AnsProlog encoding of the collapse mechanism A2.

This collapse mechanism has much in common with the mechanism A1 in terms of crack patterns: the combine mechanisms A2 is recognised if vertical cracks are identified on the façades adjacent to the VeME under observation. For this reason the AnsProlog codes, 


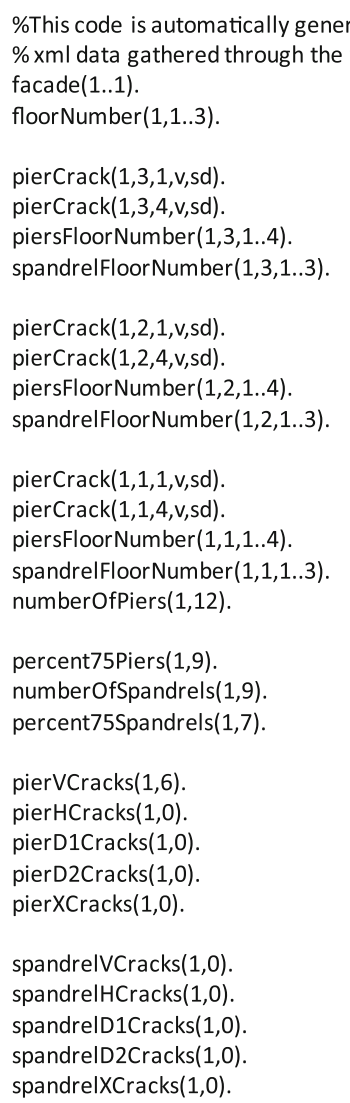

Crack data for floor level 2: vertical cracks in the first and last piers, with severe damage

Crack data for floor level 1: vertical cracks in the first and last piers, with severe damage

This block computes the number of elements

that constitute $75 \%$ of each kind.

This data is used to identify collapse mechanisms $\mathrm{H} 1$ and $\mathrm{H} 2$.

This block summarizes pier crack data for facade 1: in this casa there are 6 vertical cracks

This block summarizes spandrel crack data for facade 1 : in this case there are no cracks

Fig. 10 The encoding of the geometry and crack pattern of a single VeME (Novelli et al. 2012) gathered from the website

implemented for the collapse mechanisms A1, are reused for the collapse mechanism A2, by introducing codes which check the crack pattern not at the VeME level, but across the AA as a whole.

Furthermore, in order to encode partial and total collapse mechanisms, the crack pattern which occurs at the first floor is also encoded separately (e.g. lowpattern $\mathrm{Al}(\mathrm{Fa}, \mathrm{Fl})$ and lowpattern $\mathrm{Ar}(\mathrm{Fa}, \mathrm{Fl}))$. In order to ensure that in case the crack pattern is verified from the top the bottom of one or more VeMEs, a total collapse mechanism is identified (e.g. collapseMechanism (outOfPlaneAt (Fa, FlT, Fr), patternAa)) (Novelli et al. 2012).

The possibility in AnsProlog to implement crack patterns with codes which codify only small parts of the entire crack pattern has simplified the approach developed in the logic trees which were implemented distinctly for each collapse mechanism of Fig. 7.

With all the 18 collapse mechanisms implemented as mentioned above, the encodings of each collapse mechanism are passed to the answer set solver clingo (Gebser et al. 2007) which has the role of matching the data of the AA gathered from the website with AnsProlog codes of the collapse mechanisms in order to identify the collapse mechanism occurring on the AA in consideration.

The data recorded for the VeME of Fig. 10 reports the geometry and the damage scenario of the façade under observation, which is characterised by three floors with vertical cracks at 
patternAl(Fa,Fl) :-

pierCrack(Fa,Fl,1,v,L1),topFloor(Fa,Fl),

floorNumber(Fa,Fl), facade(Fa), damageLevel(L1).

patternAr(Fa,FI) :-rightPierFloor(Fa,FI,R),

pierCrack(Fa, Fl, $\mathrm{R}, \mathrm{V}, \mathrm{L} 2)$, topFloor(Fa, FI),

facade(Fa), floorNumber(Fa,FI), damageLevel(L2).

This rule determines whether the top left most pier/

top right most has a vertical crack. Partial crack patterns

to simulate reuse

patternAl(Fa,Fl) :-

pierCrack (Fa, FI, 1, v, L1), floorNumber( $\mathrm{Fa}, \mathrm{FlH})$,floorNumber( $\mathrm{Fa}, \mathrm{FI})$,

patternAl(Fa, $\mathrm{FIH}), \mathrm{FI}=\mathrm{FIH}-1$, facade( $\mathrm{Fa})$, damagelevel(L1). patternAr(Fa,IL) :-

pierCrack(Fa,FI,R,v,L2), rightPierFloor(Fa, FI, R),

floorNumber( $\mathrm{Fa}, \mathrm{FlH})$, floorNumber(Fa,Fl), damagelevel(L2)

patternAr(Fa, $\mathrm{FlH}), \mathrm{Fl}=\mathrm{FlH}-1$, facade(Fa).

For a given floor, if a left most/right most vertical

crack pattern was determined on the floor above,

check if this also so for the current floor

lowpatternAl(Fa, FI) :-

patternAl( $\mathrm{Fa}, \mathrm{Fl})$, not patternAl( $\mathrm{Fa}, \mathrm{FII}), \mathrm{FI}=\mathrm{FII}+1$,

floorNumber(Fa,FI),flooriNumber(Fa,Fll), facade(Fa).

lowpattern $\mathrm{Ar}(\mathrm{Fa}, \mathrm{FI})$ :

patternAr(Fa,Fl), not patternAr(Fa,Fll), $\mathrm{FI}=\mathrm{Fll}+1$,

floorNumber(Fa,FI),floorNumber(Fa,Fll), facade(Fa).

lowpatternAl(Fa,1) :- patternAl(Fa,1).

lowpatternAr(Fa,1):- patternAr(Fa,1).

lowpatternA(Fa) :-

lowpatternAl(Fa,Fl), lowpatternAr(Fa,Fll), facade(Fa),

floorNumber(Fa,FI), floorNumber(Fa,FII

collapseMechanism(outOfPlaneAt(Fa,FIT,Fr), patternA) :-

lowpatternAl(Fa, Fl), lowpatternAr(Fa, Fr), $\mathrm{Fl}<\mathrm{Fr}$, topFloor(Fa, FIT)

floorNumber(Fa,Fl), floorNumber(Fa,Flt), facade(Fa).

collapseMechanism(outOfPlaneAt(Fa,FIT,FI), patternA) :-

lowpatternAl(Fa, Fl), lowpatternAr(Fa, Fr), $\mathrm{Fl}>=\mathrm{Fr}$, topFloor $(\mathrm{Fa}, \mathrm{FIT})$,

floorNumber(Fa,FI), floorNumber(Fa,Flt), facade(Fa).

This rule determines the lowest floor on which a left/ right vertical rack was determined

Starting position to check for finding the lowest floor with continuous vertical cracks on the left/right

This rule establishes that both left and right side have a vertical cracks. It Is used the exclude other collapse mechanisms

Indicates when a collapse mechanism of PatternA occurs from the top of the facade to the floor where there is vertical crack on both sides

Fig. 11 The rules used to recognize collapse mechanism A1. (Novelli et al. 2012)

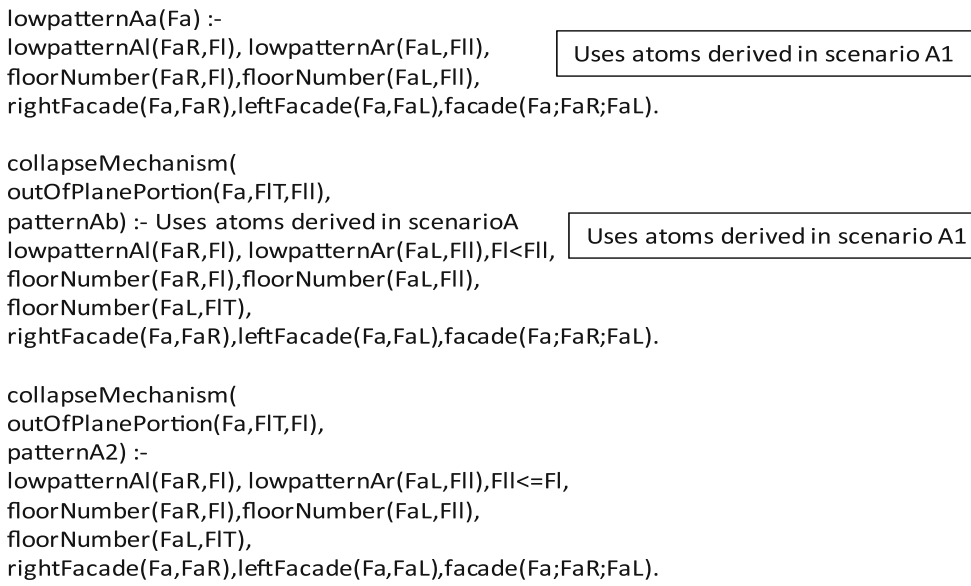

Fig. 12 The rules used to recognize collapse mechanism A2. (Novelli et al. 2012)

its edges. By passing these data into the answer set solver clingo, the result is the identification of the collapse mechanism A1.

The answer set solver clingo identifies the collapse mechanisms for an entire AA in within 1 or 2 seconds. 


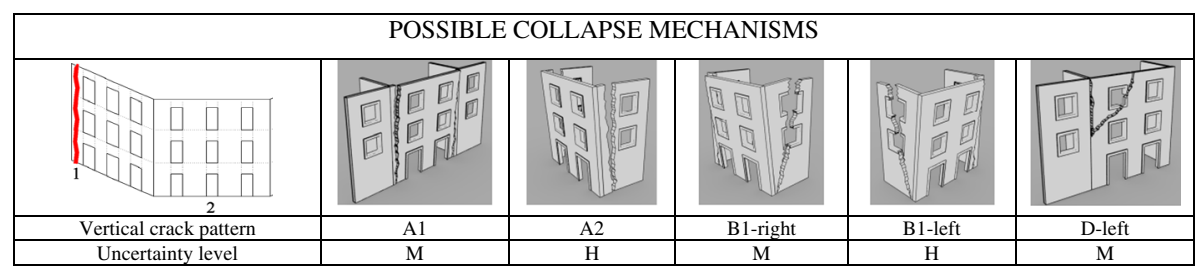

Fig. 13 Uncertainty levels of the possible collapse mechanisms identified for the vertical crack pattern recorded on the left edge of the VeME 1

Table 2 Definition of the uncertainty levels

\begin{tabular}{lll}
\hline Uncertainty & $\begin{array}{l}\text { Match between collapse mechanisms } \\
\text { (Fig. 7) predicted by LOG-IDEAH and } \\
\text { observed crack pattern }\end{array}$ & $\begin{array}{l}\text { All VeMEs, required to identify the } \\
\text { mechanism predicted by LOG-IDEAH, } \\
\text { have been inspected }\end{array}$ \\
\hline LOW & Completely & Yes \\
MEDIUM & Partially & Yes \\
HIGH & Partially & NO \\
\hline
\end{tabular}

5.3 Criteria for the identification of the uncertainty level of the collapse mechanisms

A post-earthquake assessment might be incomplete for different reasons: denied access to AAs due to lack of security, denied access to a street which does not permit to observe all VeMEs of an AA, VeMEs not visible because they are between two adjacent AAs. In all these cases of limited inspection of the AAs, it is not straightforward to identify the structural performance and consequently the failure mechanisms occurring on the AAs under consideration. However, years of experience in post-earthquake assessments (D'Ayala and Paganoni 2011; Ferreira et al. 2013; Parisi and Augenti 2013, Barbate et al. 2008, Bernardini, D’ Ayala, Modena, Speranza and Valluzzi Bernardini et al., Binda et al. 2006) have highlighted that although an AA has not been completely inspected or the observed crack pattern does not exactly match with the collapse mechanisms defined in Fig. 7, the limited collected data is still associable to more than one of them. This situation can be illustrated by way of an example.

With relation to Fig. 13, only two VeMEs of the AA have been inspected, and a vertical crack pattern has been observed on the VeME 1. This crack pattern does not completely match any of the collapse mechanisms of Fig. 7. However this can still be associated to the failure modes of Fig. 13.

In particularly, the red crack pattern partially matches A1, which occurs in case of vertical cracks on both sides of the VeME; A2, B1-right and B1-left, identifiable only in case at least three adjacent VeMes have been assessed; and D-left, although diagonal cracks are not identified.

In order to highlight whether the collapse mechanism has been captured on the basis of a total or partial match between the observed crack pattern and the mechanisms of Fig. 7, LOGIDEAH associates a level of uncertainty to each identified collapse mechanism according to the criteria reported in Table 2. 


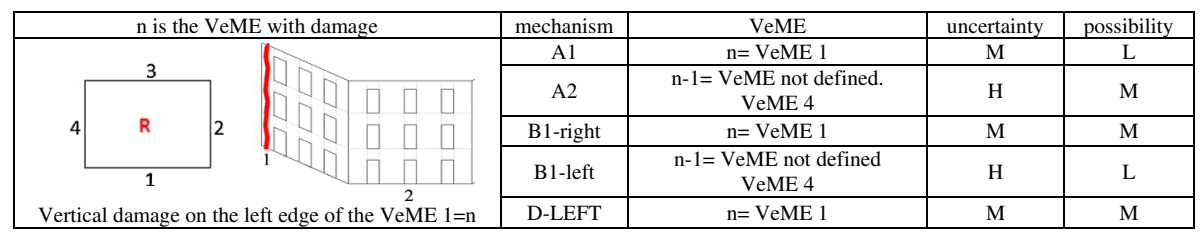

Fig. 14 Level of possibility of occurrence of the collapse mechanisms identified for the vertical crack pattern recorded on the left edge of the VeME 1

5.4 Criteria for the identification of occurrence level of the collapse mechanisms

The level of possibility of occurrence for a mechanism is higher in case the damage state of an AA is particularly severe and allows identifying the failure and the performance of the AA. Hence, if a complete inspection has been carried out on a AA, and the observed damage state points out the presence of a failure, or in other terms if the observed crack pattern exactly matches one of the mechanisms in Fig. 7, the occurrence of the mechanism is proven and the level of possibility of occurrence for the identified mechanism is high.

The real issue is to evaluate the level of possibility of occurrence in mechanisms identified through a pattern that does not exactly match the crack patterns of Fig. 7, and can map into more than one mechanism, as it illustrated in Fig. 13. Since these mechanisms might have either medium or low probability of occurrence, it is necessary to make some assumptions on the reasons why the mechanism is only partially developed, and on the state of damage of those VeMEs which have not been inspected.

The criteria developed in order to determine the level of possibility of occurrence of the mechanisms identified by the system for a crack pattern which match only partially the failure of Fig. 7 is based on considering that

1) in case of high uncertainty

a) in order to be on the conservation side, it is assumed that the unnoticed MEs are damaged and not well connected.

2) in case of medium uncertainty

a) if no damage is observed on the edges of the VeMEs, it is assumed that MEs are well connected,

b) if diagonal cracks are observed on the edges of the VeMEs, it is assumed that MEs are weakly connected,

c) if diagonal cracks are observed on the edges of the VeMEs, it is assumed that MEs are not connected.

These criteria are used by the system to evaluate the mechanism that has the higher number of verified conditions: good/bad connections between MEs or in other terms absence/presence of cracks, which increase its level of possibility of occurrence with respect to other failures identified by the system.

This situation can be illustrated by way of an example. Fig. 14 summarises the level of uncertainty and level of possible occurrence for each of the mechanisms that can be inferred by the observation of the vertical crack pattern of the AA shown in Fig. 13.

Since no damage is detected on the VeME 2, it is assumed that

- VeME 1 and 2 are well connected. 


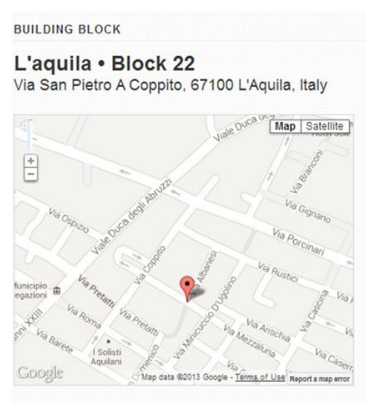

(a)

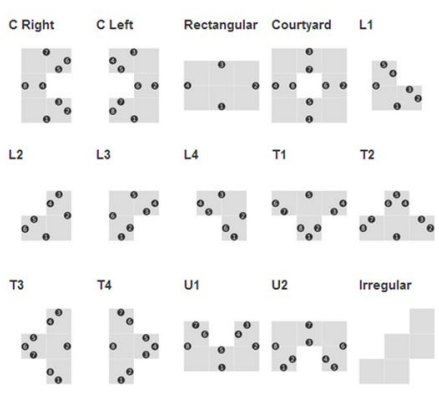

(b)

Fig. 15 Extract from the web-interface of LOG-IDEAH. a The localisation of the inspected AA block, definition of the number of AAs which belong to the AA block under investigation. b Plan geometry associable to the inspected AAs

- B1-right and A1 have medium uncertainty level. B1-right has higher possibility of occurrence with respect to A1: a failure which occurs in case of bad connection between VeME 1 and 2.

- B1-Right and D-Left have medium uncertainty and the same level of possibility of occurrence, since these mechanisms are not mutually exclusive (Shi et al. 2008).

- A2 and B1-left have high uncertainty therefore it is assumed that the weakness conditions for the VeMEs, which have not been inspected. For this reason since VeME 3 and VeME 4 have not been inspected, they are assumed not well connected. This implies that the probability of occurrence is medium and low for the mechanism A2 and B1-left, respectively.

\section{Web-interface for the data collection and data interpretation}

A web-interface available online at: http://perpetuate.cs.bath.ac.uk/ has been developed to share data in real-time with other users and to simplify the data collection. This Section is presented to provide an example of the use of the website. The urban block in Fig. 15 was inspected on site with a version of LOG-IDEAH developed for Android. The data collection of Fig. 16 has been performed for the VeMEs 22.9.1e and 22.9.4s. The AA under investigation has a corner position in the AA Block 22 (Fig. 15) that does not allow collecting data for VeMEs 22.9.2n and 22.9.3w. First LOG-IDEAH is run only for the VeME 22.9.1e and then for both VeMEs 22.9.1e and 22.9.4s. In case the only inspected VeME of the AA is the 22.9.1e, the most severe cracks are the diagonal cracks in yellow and violet and the vertical crack in red, highlighted in Fig. 17.

The two cracks describe the formation of a hinge at the bottom of 22.9.1e, which determines an overturning of the VeME. By analysing the most severe damage (with levels $\mathrm{C}$; NC; $\mathrm{SD}$ ) of 22.9.1e with LOG-IDEAH, the procedure identifies the collapse mechanism D1left, observed on site and recorded into the web browser and $\mathrm{H} 2$, which was not identified on site. Both identified failure modes by LOG-IDEAH have LOW uncertainties and HIGH possibility of occurrence, since all required VeMEs have been inspected for the capture of these mechanisms and the observed crack pattern (yellow, violet and red cracks) describes exactly the development of the identified mechanisms. 


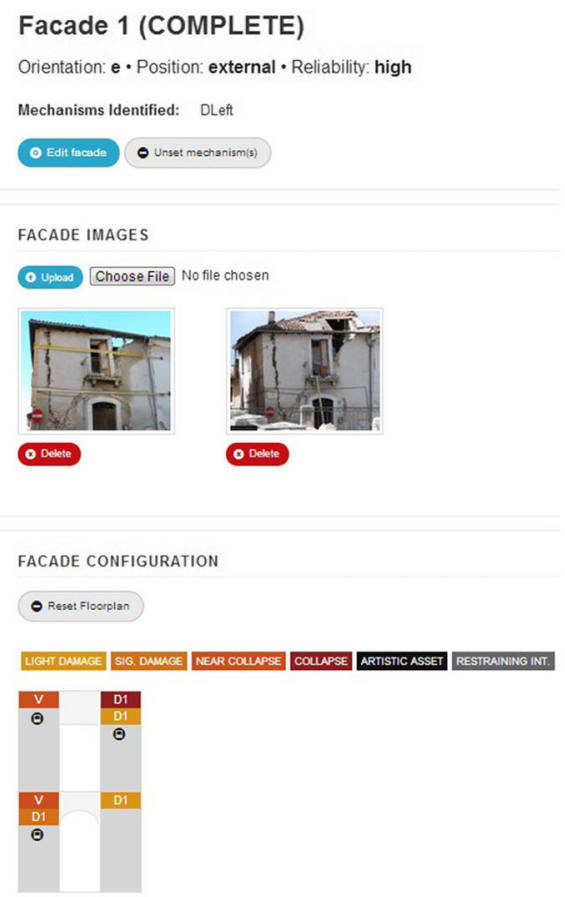

(a)

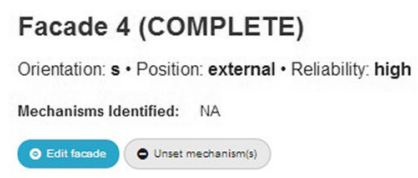

FACADE IMAGES

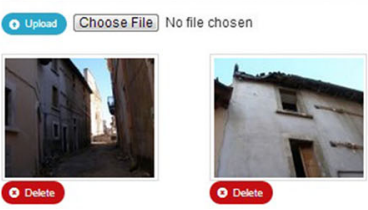

FACADE CONFIGURATION

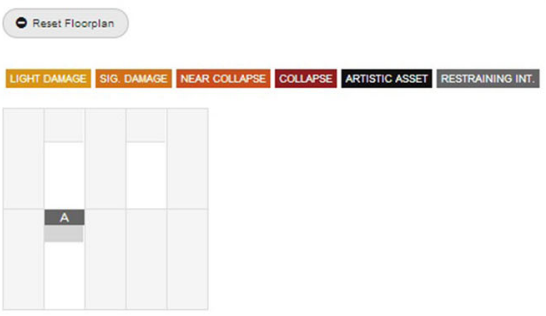

(b)

Fig. 16 Extract from the web-interface of LOG-IDEAH. a Simplified sketch and identification of the damage state of the VeME 22.9e. b Simplified sketch and identification of the damage state of the VeME 22.9s
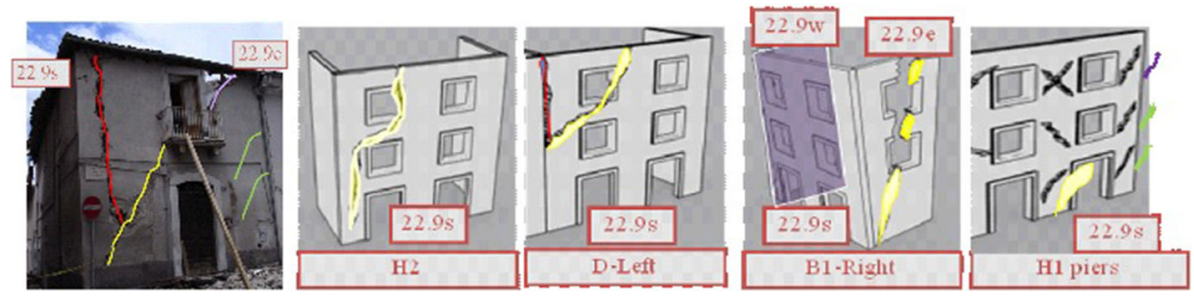

Fig. 17 LOG-IDEAH output applied to the VeME 22.9e and VeME 22.9s

In case the analysis is carried out on both VeMEs 22.9.1e and 22.9.4s, LOG-IDEAH identifies not only the collapse mechanisms D1-Left and H2 for the VeME of 22.9.1e but also B1-Right for the VeME 22.9.4s, which was not recognised on site.

As in the previous analysis, D1-Left and H2 occur with LOW uncertainty and HIGH possibility for the reasons mentioned above. B1-Right occurs with LOW uncertainty, because the identification of this mechanism derives from an incomplete survey. As for its possibility level, since 22.1.3 w has not been inspected, a bad connection between 22.1.3 $\mathrm{w}$ and 22.1.4s is assumed; therefore B1-Right has a MEDIUM possibility level.

In case the analysis is repeated by considering all levels of damage, LOG-IDEAH adds $\mathrm{H} 1$ with LOW uncertainty and HIGH possibility to the already identified mechanisms in the previous interpretation. The failure $\mathrm{H} 1$ has lower level of severity since it has been identified on the basis of cracks with light damage level. 


\section{Discussion and future work}

In the present work, LOG-IDEAH has demonstrated that expert systems are valid alternatives to the existing methodologies developed for the identification of the seismic performance of historical buildings.

In particular, since LOG-IDEAH interprets the seismic behaviour of Architectural Assets by matching observed crack patterns with predefined collapse mechanisms, new sets of rules which match crack patterns and collapse mechanisms can also be defined for building typologies different from the ones already implemented in the system.

The logic of LOG-IDEAH was initially developed in logic trees. Each logic tree describes which damage types and damage positions are required in order to identify only one specific collapse mechanism on the basis of data collected on site or by using pictures.

In order to simplify the approach used in the logic trees, a website available at (http:// perpetuate.cs.bath.ac.uk/) with password protection has been implemented in order to store geometric data and seismic data of the Architectural Assets. This data stored in XML format files is semantic tagged and transferred to an answer set solver which interprets the stored data according to set of rules, implemented in Answer Set Programming (ASP) and based on the hierarchical approach adopted in the logic trees for matching crack patterns with specific collapse mechanisms.

The encoding in ASP has allowed writing more efficient and flexible codes, by implementing the crack patterns in small distinct codes which are reused for encoding collapse mechanisms with similar crack patterns (e.g. codes written for A1 is also reused for A2). The code in ASP is much more intuitive and less error-prone than the code used in the logic trees, indeed during the process of writing the ASP a number of subtle errors, uncovered in the logic trees, were emphasised and sorted out. Moreover some particular crack patterns, which point to the identification of more than one possible mechanism, and which are hard to analyse with the only use of logic trees, were analysed and implemented directly using the ASP.

The ASP encoding of the collapse mechanisms took only few days, therefore once the rules which match crack patterns with collapse mechanisms are defined, its implementation into ASP is particularly simple.

The next step will be to extend the approach used in LOG-IDEAH for identifying the effect of pounding between Architectural Assets. Moreover, since all input data and results are georeferenced, the next version of LOG-IDEAH will have additional features incorporated, such as to download ASCI files, to upload geo-referenced data into a GIS system.

Open Access This article is distributed under the terms of the Creative Commons Attribution License which permits any use, distribution, and reproduction in any medium, provided the original author(s) and the source are credited.

\section{References}

AIS (2003) Association Colombiana de Ingegneria sismica. Manual de Campo para Inspecion De Edificio Despues de un Sismo. Manizales, Colombia

ATC-20i (2003) Applied Technology Council, user manual: mobile post-earthquake building safety evaluation data acquisition system (version 1.0). Redwood City, California

ATC-20(1989) Applied Technology Council. Procedures for post-earthquake safety evaluation of buildings. Redwood City, CA

Baral C (2003) Knowledge representation, reasoning, and declarative problem solving. Cambridge University Press, Cambridge 
Baggio C, Bernardini A, Colozza R, Corazza L, Della Bella M, Di Pasquale G, Dolce M, Goretti A, Martinelli A (2009) Manuale per la compilazione della scheda di $1^{\circ}$ livello di rilevamento danno, pronto intervento e agibilità per edifici ordinari nell'emergenza post-sismica (AeDES). Editrice Italiani nel Mondo srl - Roma

Barbat AH, Pujades LG, Lantada N, Moreno R (2008) Seismic damage evaluation in urban areas using a capacity spectrum based method: application to Barcelona. Soil Dyn Earthq Eng 28(10-11):851-865

Bernardini A, D’Ayala D, Modena C, Speranza E, Valluzzi MR (2008) Vulnerability assessment of the historical masonry building typologies of Vittorio Veneto. Special issue of Bollettino di Geofisica Teorica e Applicata, vol 49, n. 3-4, Sept-Dec, pp 463-484

Binda L, Cardani G, Saisi A, Valluzzi MR (2006) Vulnerability analysis of the historical buildings in seismic area by a multilevel approach. Asian J Civil Eng (Building and Housing) 7(4):343-357

Bosiljkov V, Gostic S, Krzan M, Antolinc D, Žarnic R (2009) Efficient models for the assessment of vulnerability of historical monuments in case of armed conflicts (in Slovenian). Report for CRP MIR M4-0207

Brain M, Cliffe O, De Vos M (2009) A pragmatic programmer's guide to answer set programming. In: Software Engineering for answer set programming (SEA09), 2009-09-14, Potsdam

Cadei M, Lazzari M, Salvaneschi P (1990) Safety management of civil structures using knowledge based systems. In: Proceedings of the third international conference on industrial and engineering applications of artificial intelligence and expert systems—volume 2 (IEA/AIE '90), 1990

Carreno ML, Cardona OD, Barbat AH (2004) Expert system for building damage evaluation in case of earthquake. In: 13th world conference on earthquake engineering. Vancouver, BC, Canada, 1-6 Aug 2004, paper no. 3047

CEN (2005) Eurocode 8: design of structures for earthquake resistance. Part 3: strengthening and repair of buildings, CEN-EN 1998-3

Centro Nacional de Prevención de Desastres (CENAPRED). Norma para la Evaluación del Nivel de Daño por Sismo en Estructuras y Guía Técnica de Rehabilitación (Estructuras de Concreto Reforzado). Cuadernos de Investigación, Número 37, México, (1996)

Chen SY, Zheng Y, Cattani C, Wang W (2012) Modelling of biological intelligence for SCM system optimization. Comput Math Methods Med, vol 2012, Article ID 769702, p 30

Chitta B (2003) Knowledge representation, reasoning, and declarative problem solving. Cambridge University Press, Cambridge 2003

Corbane C, Saito K, Dell'Ora L, Bjorgo E, Gill SPD, Piard BE, Huyck CK, Kemper T, Lemoine G, Spence RJS, Shankar R, Senegas O, Ghesquiere F, Lallemant D, Evans GB, Gartley RA, Toro J, Ghosh S, Svekla WD, Adams BJ, Eguchi RT (2011) A comprehensive analysis of building damage in the 12 January 1020 Mw7 Haiti earthquake using high-resolution satellite- and aerial imagery. Photogramm Eng Remote Sens 77(10):997-1009

D'Ayala D, Novelli V (2012a). Formulation of vulnerability models including the survey form to collect the data re-quired by the adopted models. Perpetuate project

D'Ayala D, Novelli V (2012b). Deliverable 30: freeware software with the implementation of the vulnerability models. Perpetuate project

D'Ayala D, Paganoni S (2011) Assessment and analysis of damage in L'Aquila historic city centre after 6th April 2009. Bull Earthq Eng 9(1):81-104

D'Ayala D, Novelli V (2010) Deliverable D5: abacus of the most common seismic damage. Perpetuate project

D'Ayala D, Speranza E (2003) Definition of collapse mechanisms and seismic vulnerability of historic masonry buildings. Earthq Spect, vol 19, Aug, pp 479-509

D'Ayala D (2005) Force and displacement based vulnerability assessment for traditional buildings. Bull Earthq Eng, vol 3, Springer, pp 235-265

Dell'Acqua F, Bignami C, Chini M, Lisini G, Polli D Stramondo S (2011) Earthquake damages rapid mapping by satellite remote sensing data: L'Aquila April 6th, 2009 event. IEEE Journal of volume: 4, pp(s) 935-943

De Vent IAE, Van Hees RPJ, Hobbelman GJ (2008) Towards a systematic diagnosis of structural damage. SAHC 2008, Bath, Uk

D.P.C.M. 23 February (2006) (G.U. 7.3.2006, n. 55)

De Vos M, Kiza D, Oetsch J, Pührer J, Tompits H (2012) Answer-set programs in lana. Theory and Practice of Logic Programming, 2012

Dovers SR, Handmer JW (1995) Ignorance, the precautionary principle, and sustainability. Ambio Stockholm [AMBIO] 24(2):92-97

Eiter T, Leone N, Mateis C, Pfeifer G, and Scarcello F (1998) The KR system dlv: Progress report, comparisons and benchmarks. In: Proceedings of the 6th international conference on principles of knowledge representation and reasoning (KR1998), pp 406-417. Morgan Kaufmann

Ferreira TM, Vicente R, da Silva JARM, Varum H, Costa A (2013) Seismic vulnerability assessment of historical urban centres: case study of the old city centre in Seixal, Portugal. Bull Earthq Eng 11(5):17531773 
Gebser M, Kaufmann B, Neumann A, Schaub T (2007) Conflict-driven answer set solving. In: Proceedings of the 20th international joint conference on artificial intelligence (IJCAI 2007). AAAI Press/The MIT Press, 2007, pp 386-392

Gelfond M, Lifschitz, V (1988) The stable model semantics for logic programming. In: Proceedings of the 5th international conference and symposium on logic programming. MIT Press, 1070:1080

Gelfond M, Lifschitz V (1991) Classical negation in logic programs and disjunctive databases. New Gener Comput 9(3-4):365-386

Gomes CP, Kautz H, Sabharwal A, Selman B (2008) Satisfiability solvers. Handbook of knowledge representation 89. Chapter 2:89-134

Goretti A, Di Pasquale G (2002) An overview of post-earthquake damage assessment in Italy. Eeri invitational workshop. An action plan to develop earthquake damage and loss data protocols, California

Hancilar U, Tuzun C, Yenidogan C, Erdik M (2010) ELER software: a new tool for urban earthquake loss assessment. Nat Haz Earth Syst Sci 10:2677-2696

Hassanzadeha R, Nedović-Budića Z, Alavi Razavib A, Norouzzadehb M, Hodhodkianb H (2012) Interactive approach for GIS-based earthquake scenario development and resource estimation (Karmania hazard model). Comput Geosc, volume 51, February 2013, pp 324-338

Hiroo K (2005) Real-time seismology and earthquake damage mitigation. Ann Rev Earth Planet Sci 2005(33):195-214. doi:10.1146/annurev.earth.33.092203.122626CopyrightcbyAnnualReviews

Hiroyuki M, Saburoh M, M.EERI (2006) Updating GIS building inventory data using high-resolution satellite images for earthquake damage assessment: application to Metro Manila, Philippines. Earthquake Spectra, volume 22, No 1, pp 151-168, February 2006, Earthquake Engineering Research Institute

Hoffmann J (2007) Mapping damage during the Bam (Iran) earthquake using interferometric coherence. Int J Remote Sens 28(3):1199-1216

Kanamori H (2005) Real-time seismology and earthquake damage mitigation. Ann Rev Earth Planet Sci 33:195-214. doi:10.1146/annurev.earth.33.092203.122626

Lang DH, M.EERI, Gutiérrez FVC (2010) RISe: illustrating geo-referenced data of seismic risk and loss assessment studies using Google Earth. J Earthq Spect, vol 26, no 1, 2010. doi:10.1193/1.3283906

Lagomarsino S, Penna A, Galasco A, CAttari S (2013) TREMURI program: an equivalent frame model for the nonlinear seismic analysis of masonry buildings. Eng Struct 56:1787-1799

Lagomarsino S, Abbas N, Calderini C, Cattari S, Rossi M, Gianni Corradini R, Marghella G, Mattolin F, Piovanello V (2011) Classification of the cultural heritage assets and seismic damage variables for the identification of performance levels. In: Proceedings of the structural repairs and maintenance of heritage architecture conference (STREMAH). Wit Press, pp 697-708, ISSN 1743-3509

Li Y, Chen G, Wang B, Zheng L, Zhang Y, Tang C (2013) A new approach of combining aerial photography with satellite imagery for landslide detection. Nat Haz 66(2):649-669 March 2013

Melchor-Lucero O, Ferregut C (1995) Toward an expert system for damage assessment of structural concrete elements. Artif Intel Eng Des Anal Manufact 9:401-418

Molina S, Lang DH, Lindholm CD (2010) ELENA: an open-source tool for seismic risk and loss assessment using a logic tree computation procedure. Comput Geosci 36(2010):257-269. doi:10.1016/j.cageo.2009. 07.006

Novelli V, D’Ayala D (2011) Seismic damage identification of cultural heritage assets. Conference of seismic protection of cultural heritage. Antalya, Turkey

Novelli V, D’Ayala D, De Vos M, Padget J, (2012) LOG-IDEAH: ASP for Architectural Asset Preservation. In: 28th international conference on logic programming

Maruyama Y, Yamazaki F, Matsuzaki S, Miura H, Estrada M (2012) Evaluation of building damage and tsunami inundation based on satellite images and GIS data following the 2010 Chile earthquake. Earthq Spect 28(S1):S165-S178 June 2012

O.P.C.M n. 3753, Gazzetta Ufficiale, 7th April (2009), n 81 (2) (in Italian)

Ogawa H, Fu KS (1985) An inexact inference for damage assessment of existing structures. Int J Man Mach Stud 22:295-306

Papa F, Zuccaro G (2004) Medea: a multimedia and didactic handbook for seismic damage evaluation. European seismological commission XXIX general assembly. University and GFZ Potsdam, Germany

Parisi F, Augenti N (2013) Earthquake damages to cultural heritage constructions and simplified assessment of artworks. Eng Fail Anal 34:735-760

Pengzhen L, Shengyong C, Yujun Z (2012) Artificial intelligence in civil engineering. Academic editor: Fei Kang, Hindawi Publishing Corporation. Mathematical problems in engineering, volume 2012, Article ID 145974, p 22. doi: 10.1155/2012/145974

Pollino M, Fattoruso G, La Porta L, Della Rocca AB, James V (2012) Collaborative open source geospatial tools and maps supporting the response planning to disastrous earthquake events. Future Internet 4:451-468. doi:10.3390/fi4020451 
Serra EMT, Delouis B, Emolo A, Zollo A (2013) Combining strong-motion, InSAR and GPS data to refine the fault geometry and source kinematics of the 2011, Mw 6.2, Christchurch earthquake (New Zealand). Geophys J. doi:10.1093/gji/ggt186

Shi Y, D'Ayala D, Prateek J, (2008) Analysis of out-of-plane damage behaviour of unreinforced masonry walls. In: 14th international brick and block masonry conference, 2008-02-17-2008-02-20, Sydney

Tong XH, Liu SJ (2009) Geometric processing of QuickBird stereo imageries for urban land use mapping: a case study in Shanghai, China. IEEE J Select Topics Appl Earth Observ Remote Sens 2(2):61-66

Turker M, Cetinkaya B (2005) Automatic detection of earthquake-damaged buildings using DEMs created from pre- and post-earthquake stereo aerial photographs. Int J Remote Sens 26(4):823-832

Vatan M, Görün A (2011) Pre-hazard evaluation criteria for risk assessment of masonry monumental historic structures. In: WCCE-ECCE-TCCE joint conference 2-seismic protection of cultural heritage

Wielanda M, Pittorea M, Parolaia S, Zschaua J, Moldobekovb B, Begalievc U (2012) Estimating building inventory for rapid seismic vulnerability assessment: Towards an integrated approach based on multi-source imaging. Soil Dyn Earthq Eng, volume 36, May 2012, pp 70-83

Wuwongse V, Asian Inst. of Technol in Bangkok, Thailand, Anutariya C, Akama K, Nantajeewarawat E (2001) XML declarative description: a language for the semantic web intelligent systems. IEEE (volume:16, Issue: 3), pp (s): 54-65; ISSN: 1541-1672

Zuccaro G, Papa F (2007) Manual for earthquake damage evaluation and safety assessment. CD ROM 